\title{
Pfadfinder.
}

\section{Zur Kolonialisierung des}

\section{geographischen Raumes}

Alexander Honold

\section{Raum als Ressource}

In den kolonialen Systemen des späten I9. Jahrhunderts waren Menschen und Handlungen von kulturell determinierten Konstruktionen des Raumes und der Raumerfahrung bestimmt. Gemäß dem Selbstbild der kolonialen Akteure, die hierin das Erbe des Zeitalters der Entdeckungen anzutreten vermeinten, war der Ausgriff europäischer Nationen auf überseeische Weltregionen durch ein Zusammenspiel von Bewegungsenergie und scheinbar unausgeschöpften Reserven an Raum gekennzeichnet. Der geographisch erfasste Raum als solcher wurde nun zu einer primären Ressource. Hatte man sich in den kolonialen Projekten der Frühen Neuzeit mit ihrem Missionsauftrag zur Jagd nach den sagenhaften Bodenschätzen El Dorados ermächtigt gefühlt, so traten im beginnenden Industriezeitalter mit seinen bevölkerungspolitischen Krisenschüben andere Motive in den Vordergrund, etwa die Suche nach Rohstoffgebieten und neuen Siedlungsräumen. Dabei blieben pragmatische und imaginäre Ziele eng miteinander verbunden, etwa in der fast magischen Ausstrahlungskraft der Weltkarte. Mit ihren klangvollen exotischen Namen, dem bizarren Gewirr von Küstenlinien, Gebirgs- und Flussläufen und dem gleichförmigen Gradnetz, das in gleichsam spielerischer Ordnungsliebe über die Kontinente gespannt war, glich die kartographische Repräsentation der »Welt« selbst schon einem gewaltigen Aufmarschgebiet für künftige Abenteurer. In Joseph Conrads Heart of Darkness erinnert sich Kapitän Marlow seines kindlichen Faibles für imaginäre Reisen durch den kartierten Raum. »Now when I was a little chap I had a 
passion for maps. I would look for hours at South America, or Africa, or Australia and lose myself in all the glories of exploration. « ${ }^{\mathrm{I}}$

Unverkennbar - und anhand eigener träumerischer >Finger-Reisen subjektiv durchaus nachvollziehbar - hat der von der Landkarte aufbereitete Raum eine suggestive affordance; er offeriert pflegeleichte Besitztümer und fordert zu global dimensionierten Reiseplänen auf. Besondere Sogkraft ging dabei noch zu Conrads Zeiten von jenen berühmten »weißen Flecken« aus, die zwar Gebiete jenseits der bereits in Besitz genommenen Welt anzeigten - unzugängliche Hochgebirge, Trocken- und Eiswüsten, Dschungel- und Steppenlandschaften -, jedoch schon durch ihre Einbindung in die universelle territoriale Ordnung einer Ökonomie der Knappheit unterworfen waren. Schon Herder hatte in seinen Ideen zur Philosophie der Geschichte der Menschheit einen mit dem Vordringen der Zivilisation unaufhaltsam einhergehenden Schwundprozess für solche Rückzugsgebiete des Fremden prognostiziert. »Je mehr die Kultur der Länder zunimmt, desto enger wird die Wüste; desto seltner ihre wilden Bewohner.«² Unter dem Eindruck der spektakulären Weltumseglungen Cooks, Bougainvilles und anderer ließ sich die fortschrittsgewisse Geschichtsphilosophie der Aufklärung ohne größere Friktionen in eine spatiale Ordnung übertragen. Enzүklopädie und Circumnavigation waren korrespondierende Unternehmungen auf dem Weg zu einer allumfassenden, erdumspannenden Totalität des Wissens, bei der sich alle Differenzen und Devianzen zu einem gemeinsamen Tableau der Orte und Arten fügen lassen sollten. Dem Ideal ganzheitlicher Anschauung war auch der Naturforscher Alexander von Humboldt verpflichtet, dessen Reisen zugleich den Übergang vom Universalgelehrtentum der Goethezeit zur fachdisziplinären Spezialisierung des positivistischen Zeitalters markierten. ${ }^{3}$ Verbürgte die tatsächliche Fortbewegung auf Reisen eine im Wortsinne »ästhetische« Erfahrung der Landschaft und ihrer kulturellen Prägungen, so lieferte die Empirie naturkundlicher Messungen »ungeheure

1 I Joseph Conrad: Heart of Darkness (I902), London I994, S. II.

2 I Johann Gottfried Herder: Ideen zur Philosophie der Geschichte der Menschheit, hg. von Martin Bollacher, Frankfurt am Main I989, S. 233.

3। Den Versuch einer Vermittlung beider Tendenzen unternimmt Humboldt in seinem Hauptwerk Kosmos, das er im Wortsinne >welt-anschaulich »Die Geschichte der physischen Weltanschauung ist die Geschichte der Erkenntnis eines Naturganzen, die Darstellung des Strebens der Menschheit, das Zusammenwirken der Kräfte im Erd- und Himmelsraum zu begreifen« (Alexander von Humboldt: Kosmos. Entwurf einer physischen Weltbeschreibung, 2 Bde., Darmstadt I993, Band 2, S. 88; vgl. ebd., S. 94). Im Rekurs auf das mit dem Begriff »Kosmos« aufgestellte Prinzip der Schönheit des Wohlgeordneten ließ sich das sinnlich-ästhetische Verhältnis zur Natur, wie es seit der Aufklärung von Philosophie und Dichtung ausgearbeitet wurde, mit dem seither schnell zunehmenden positiven Faktenwissen versöhnen, so der Grundgedanke des humboldtschen Naturbildes. 
Datenmengen«, denen sich ein akribischer Faktensammler wie Humboldt, so Gernot und Hartmut Böhme, »in einem fast süchtigen Ausmaß [...] ausgesetzt « habe. ${ }^{4}$

Mit Humboldt als einem ihrer Gründerväter ist dieser Zwiespalt für die Entwicklung der modernen Geographie im I9. Jahrhundert bestimmend geblieben. In der neuen Disziplin überlagerten sich die tradierten Kenntnisbestände der mathematischen, physikalischen und geo-chemischen Erdwissenschaften mit einem eher amorphen Betätigungsfeld kultureller Neugier. Zur Mitte des I9. Jahrhunderts schien Geographie, als reflektierte Reise- und Beschreibungskunst, eher die Domäne der Feuilletons als die der Universitäten zu werden. Journalistische Reiseschriftsteller und Romanautoren wie Humboldts Ziehsohn Balduin Möllhausen verwandelten das Wissen über ferne Länder und Menschen in einen populären und einträglichen Lesestoff, während sich die in Deutschland eher langsam voranschreitende Institutionalisierung einer >wissenschaftlichen< Geographie, ganz nach dem französischen Vorbild der Revolutionszeit, überwiegend an militärtechnischen Erfordernissen ausrichtete. ${ }^{5}$

Die imaginativen Anteile des geographischen Wissens und die damit verbundenen sozialen Wunschenergien konnten sich im letzten Jahrhundertdrittel gerade dort behaupten und vermehren, wo im Zuge der kolonialen Expansion Grenzlinien verschoben und Territorien einer Neuordnung unterzogen wurden. Als kulturelle Formation beerbt der Kolonialismus insofern das mit den Künsten und Wissensformen der >Erdbeschreibung verbundene Faszinationsmoment fremder Welten und abenteuerlicher Reisen. Neben und vor der Kolonialherrschaft im politischen Sinne sind die ko-

4 | Gernot Böhme/Hartmut Böhme: Feuer - Wasser - Erde - Luft. Eine Kulturgeschichte der Elemente, München I996, S. IIf.

5 | Die disziplinäre und akademische Etablierung der Geographie war eine preußische und vorrangig militärisch motivierte Initiative. Der 1820 an die Berliner Universität berufene erste deutsche Lehrstuhlinhaber für physische Geographie, Carl Ritter (I779-I859), versah neben seinem Lehramt noch eine Dozentenstelle an der Berliner Allgemeinen Kriegsschule. Erst fünfzehn Jahre nach Ritters Tod wurde die Stelle I874 mit Heinrich Kiepert (I8I8-1899) wieder besetzt, dessen historische Auslegung des Faches I886 durch die Berufung des für physische Geographie zuständigen Ferdinand von Richthofen (I833-I905) kompensiert wurde (Lothar Zögner: »Geographie«, in: Tilmann Buddensieg/Kurt Düwell/Klaus-Jürgen Sembach [Hg.], Wissenschaften in Berlin, Berlin I987, Band 2, S. 85f.). »Die Richtlinien des preußischen Kultusministers Adalbert Falk zur Gründung geographischer Lehrstühle im Jahre I874 führten dazu, daß sämtliche preußische Universitäten, die bis dahin nicht über einen Geographielehrstuhl verfügten, in rascher Folge bis Ende der siebziger Jahre Ordinariate erhielten« (Marita Baumgarten: Professoren und Universitäten im I9. Jahrhundert, Göttingen I997, S. 88). 
lonialen Imaginationen verknüpft mit elementaren Szenarien der Raumerschließung und -bemächtigung. Diese erfolgten meist und mit großer Selbstverständlichkeit in einem Raum, der sich den europäischen Ankömmlingen - ungeachtet seines tatsächlich vorhandenen Besiedlungsgrades - als in die Weite hingebreitetes Niemandsland darbot. Der koloniale Raum war scheinbar unbesiedelt, aber eben darum keineswegs kulturell unbearbeitet. Raum bzw. Räumlichkeit war keine neutrale, politisch und moralisch indifferente Größe. ${ }^{6}$ Im Gegenteil: Gerade die für den Kolonialismus nützliche Arbeitsfiktion des leeren Raums, des »Raums ohne Volk«, wie man in Umkehrung der berüchtigten Formel Hans Grimms sagen könnte, 7 ist ein Beispiel für die diskursive und epistemologische Verfasstheit selbst der elementarsten Wahrnehmungskategorien. Die phänomenale Evidenz dieses (kulturell produzierten) leeren Raumes war ein wichtiger Bestandteil des Kolonialisierungsprozesses und begründete zugleich den Mythos, mit dem die Kolonisatoren ihre historische Mission zu legitimieren suchten. ${ }^{8}$ An diesem Prozess waren wissenschaftliche, aber auch literarästhetische Darstellungsmuster beteiligt, deren Zusammenspiel zu einem proto-kolonialen Raumszenario im Folgenden anhand einiger Beispiele aus der Vorgeschichte der kolonialen Landnahme näher beleuchtet werden soll.

\section{Horizontflucht: Der Zug der Karawane}

In Alexis de Tocquevilles großer Abhandlung Über die Demokratie in Ameri$k a$ findet sich die Bemerkung, dass die Entvölkerung des Himmels den Blick auf Flüsse und Berge gelenkt habe, woraus »im letzten Jahrhundert die Dichtung« entstand, »die man im eigentlichen Sinne die beschreibende

6 I Dies hat John Noyes am diskursiven Material des Kolonialismus von Deutsch-Südwestafrika eindrucksvoll zeigen können: »Colonial landscape is not found by the colonizer as a neutral and empty space, no matter how often he assures us that this is so. This is one of the most persistent myths of colonization « (John Noyes: Colonial Space. Spatiality in the Discourse of German South West Africa I884-I9I5, Chur u.a. I992, S. 6). Die in den Raum ausgreifende, raumerschließende Funktion des Kolonialen ist für den deutschen Kontext verschiedentlich betont worden, zuletzt von Russell Berman, der mit einem gewissen Seitenhieb gegen ethnozentrierte Varianten der Kolonialismuskritik feststellt, das tragende Konzept für die Analyse zumindest des deutschen Kolonialprojektes sei »space, not race« (Russel Berman: Enlightenment or Empire. Colonial Discourse in German Culture, Lincoln, London I998, S. 3).

7 | Hans Grimm: Volk ohne Raum, München I934.

8| Robert D. Sack: Conceptions of Space in Social Thought. A Geographic Perspective, London I980. 
genannt hat «. ${ }^{9}$ Den Zusammenhang, den Tocqueville damit nahe legt, kann man nun so auffassen, dass erst mit der säkularen Aufwertung des Diesseitigen und Irdischen auch die Landschaftsbeschreibung als ein literarischer Gegenstand denkbar und akzeptabel wurde. Doch liegt in dieser neuzeitlichen Dignität des Bodens, der Landschaft und des Horizontalen auch ein unbestreitbares Moment von Zwang. Die scharf gezogene Grenze zum Himmlisch-Metaphysischen bannt die Höhenflüge des Geistes, fesselt sie an die Bedingungen der Schwerkraft und den Radius ihres Gesichtskreises. Darin deutet sich ein verwunderlicher Befund an: dass nämlich das kulturelle Wissen der Geographie die Erde als Oberfläche behandelt. Die koloniale Struktur des geographischen Raumes ist Indikator einer Anschauungsweise, bei der die Erde wieder zur Scheibe wird. Die Randlinie des Horizonts, die den geographischen Alltagsverstand begrenzt, ist das Ergebnis eines jahrhundertelangen, aber dramatischen säkularen Prozesses der Entgötterung, der Tilgung des Heiligen von der Erde. ${ }^{\text {IO }}$ Wenn die Götter oben bleiben, wird der irdische Raum zu einem Gefilde der Pertinenz; was der Horizont umschließt, ist prinzipiell zugänglich. Das wiederum bedeutet: Es gibt keine »Topographie des Übergangs« mehr, wie sie etwa Kolumbus noch vor Augen hatte, als er die Inseln der Seligen ansteuerte, die er auf der Ostseite Indiens vermutete. ${ }^{\text {II }} \gg$ Nach einem langen Prozeß der Desintegration«, so Albrecht Koschorke in seiner Geschichte des Horizonts, »zerfällt die Vorstellung eines paradiesischen Ortes auf der Erde in zwei Komponenten: ins Fabulistische und ins Koloniale. ${ }^{\mathrm{I2}}$ Oder beides verbindet sich in der Textproduktion des Fabulistisch-Kolonialen.

Die Bewegung zum Horizont hin entspringt der Einsicht in eine grundlegende Mangelstruktur der Diesseitigkeit. Es muss, wenn die vertikale Erlösung suspendiert ist, immer so fortgehen: das Leben, die Reise, die Schrift.

9 | Alexis de Tocqueville: Über die Demokratie in Amerika, 2 Bde., Zürich I987, Band 2, S. Io8f.

$10 \mid$ |Der intensive Verkehr zwischen Gottwelt und Menschenwelt am Berührungsrand von Himmel und Erde, von dem die Mythen erzählen, fällt einer theologischen Abhebung der Ebenen zum Opfer. [...] Die Erdfläche wird vom Heiligen gereinigt, die Vorstellung des Himmelsreichs dagegen bedient sich des überkommenen Anschauungsvorrats nur noch in uneigentlicher Form « (Albrecht Koschorke: Die Geschichte des Horizonts. Grenze und Grenzüberschreitung in literarischen Landschaftsbildern, Frankfurt am Main I990, S. 22).

$11 \mid »[\ldots]$ das Beispiel zeigt, daß sich hier die Paradiesvorstellung ganz von ihrem religiösen Mutterboden, der christlichen Eschatologie, abgelöst hat und einer veränderten, ihrer gesellschaftlichen Basis nach frühkapitalistischen Grammatik des Denkens und der Wünsche unterliegt« (A. Koschorke: Geschichte des Horizonts, S. 2I).

12 | A. Koschorke: Geschichte des Horizonts, S. 22. 
Sinnbild dieser perpetuierten Bewegung ist die Horizontlinie selbst, als eine Grenzlinie, die zwar jederzeit angepeilt, aber niemals überschritten oder auch nur erreicht werden kann. Mit dem Horizont etabliert die Neuzeit eine neue Art von geographischer Ferne, die nun nicht mehr als Schwelle zum Anderen fungiert, etwa zu den sagenhaften Monstra des Erdrandes, sondern auf die endlose Prozessualität des Reisens selbst verweist. ${ }^{13}$ Die Horizontlinie verbindet zwei geographische Phänomene, die wir üblicherweise für kontradiktorisch halten: Grenze und Transgression. ${ }^{\mathrm{I}}{ }^{4}$ Als medialer Effekt korrespondiert der Horizont mit der neuzeitlichen Errungenschaft der Zentralperspektive, denn in ihr fungiert das Tafelbild nicht mehr als greifbarer Raum, sondern als ein Fenster, das sich in der Mitte zwischen Sehstrahl und Fluchtpunkt öffnet und dadurch zur puren Sogwirkung wird. ${ }^{\text {I5 }}$

Die Dynamik des Horizontalen verbündet sich mit der expansiven Tendenz des modernen Unbehagens ${ }^{16}$ am Hier und Jetzt zur unendlichen Progression. Für den Reisenden gibt es, wie einst für den Pilger, kein irdisches und kaum ein jenseitiges Ziel. Was er durchquert, ist im bildhaften Sinne stets nur Perspektive, im moralischen Verstande hingegen Wüste, die puritanische Ödnis einer innerweltlichen Askese, die das Innehalten und Genießen niemals verlohnt. ${ }^{17}$

$13 \mid$ | Zusammenfassend kann gesagt werden, daß die Nation der Raumgrenze in einer von Nikolaus von Kues ausgehenden und bis zu Newton führenden Tradition eine Art logischen und bildlichen Transgressionszwang auslöst, in dessen Folge jede Umkehrung des Blicks, in der Form jenes mythischen Vermögens, die Welt von außen zu betrachten, unmöglich wird (A. Koschorke: Geschichte des Horizonts, S. 40; Hervorhebung im Original).

14 Auch im Kolonialen ist dieser Widerspruch als Ambivalenz wirksam. »The increasingly rigid organization of space in colonization is constantly threatening to fragment the totality of space it seeks to create. To counter this, colonial discourse develops a mythic function - it creates an unlimited mobility across boundaries, and in doing so, it reconfirms these boundaries within a totalized experience of space« (J. Noyes: Colonial Space, S. 20).

15 | Vgl. A. Koschorke: Geschichte des Horizonts, S. 70.

16 | John Locke beantwortet die Frage, »was es eigentlich ist, das den Willen hinsichtlich unserer Handlungen bestimmt«, mit der Annahme, »daß es nicht, wie man gewöhnlich annimmt, das in Aussicht stehende, größere Gut ist, sondern irgendein (und zwar meist das drückendste) Unbehagen, das man gegenwärtig empfindet. [...] das Begehren ist ein Unbehagen des Geistes, das durch den Mangel eines abwesenden Gutes verursacht wird « (John Locke: Versuch über den menschlichen Verstand, Hamburg I98I, S. 300).

17 | Vgl. Zygmunt Bauman: »Vom Pilger zum Touristen«, in: Das Argument 205 (1994), S. 389-408. 
In schauerlichen Nächten,

Bedrohet von Gefechten;

Das Stöhnen der Kamele

Durchdrang das Ohr, die Seele,

Und derer die sie führen

Einbildung und Stolzieren.

Und immer ging es weiter

Und immer ward es breiter

Und unser ganzes Ziehen

Es schien ein ewig Fliehen,

Blau, hinter Wüst und Heere,

Der Streif erlogner Meere. ${ }^{18}$

Diese Zeilen entstammen einem jener Gedichte, mit welchen Goethe den starken Eindruck zu parieren versuchte, den die arabische Lyrik aus islamischer und vorislamischer Zeit bei ihm hinterlassen hatte. ${ }^{\text {I9 }}$ Goethes Beduinendichtung, die vermutlich im Februar I8I5 entstand und zunächst unter dem Titel Karawane verzeichnet ${ }^{20}$ wurde, inszeniert den Schwindel des je zurückweichenden Horizonts und das Phantasma des leeren Raumes immer weiter, immer breiter - anhand einer Fortbewegungsart, die zugleich eine soziale Gesellungsform ist. Die Karawane meint beides, den $\mathrm{Zu}$ sammenschluss der Beduinen zu einem gemeinsamen Zug und die dabei gewählte Bewegungsform, die als nomadische zum Inbegriff einer ganzen Existenzweise wird. Der Topos der Wüste ${ }^{2 \mathrm{I}}$ evoziert dabei nicht so sehr die Vorstellung eines lebensfeindlichen, vegetationslosen Gebietes, sondern vielmehr die eines Raumes, der von der unerreichbaren, trügerischen Verheißung wasserspendender Quellen phantasmagorisch aufgeladen ist. Der

18 I Johann Wolfgang Goethe: West-östlicher Divan, Berliner Ausgabe, Band III, Berlin, Weimar I979, S. $58 \mathrm{f}$.

19 | Vorbild war in diesem Falle die vorislamische Beduinendichtung der so genannten Moallakat, einer Anthologie aus dem 6. Jahrhundert. Katharina Mommsen (Goethe und die arabische Welt, 2. Aufl., Frankfurt am Main I989) erwähnt eine Eintragung in Goethes Tagebuch vom 23. Februar I8I5 (>[Vormittags] Moallakats Beduinen Zustand ) und folgert daraus: »Die Wahrscheinlichkeit ist groß, daß aus dieser Situation jenes Gedicht erwuchs, das im Divan eine ins Breite gehende Schilderung des Beduinenmilieus darbot« (S. 95; vgl. auch S. 51).

20 | Im Wiesbadener Register jener Gedichte, die auf die Lektüre der arabischen Dichtung reagieren (vgl. K. Mommsen: Goethe und die arabische Welt, S. 95).

21 | Max Rychner kommentierte: »Das Gedicht vermittelt dem Divan den Wüstenaspekt, den der Härte, des Dürren, Empfindungslosen und dennoch beklemmend Großartigen« (J.W. Goethe: West-östlicher Divan, Zürich I952, S. 464). 
Zug, das Ziehende, der ewig lockende Streif am Horizont sind hier als einander bedingende, interagierende Phänomene gestaltet.

Die nomadische Haltung der Beduinen interessierte Goethe nicht nur als exotisches Sujet, sondern auch als ein Extremfall des Zusammenspiels von Raum, Bewegung und kultureller Lebensweise. Dies lässt sich unter anderem daran ablesen, dass Goethe die Motive des Nomadischen nach der Divan-Zeit auch den ihn seit I807 beschäftigenden Wanderjahren überschrieben hat. Dort geht es freilich nicht um die elegische Verherrlichung einer ursprünglichen, nomadischen Freiheit, wie sie etwa in Hölderlins $\mathrm{Hy}$ perion um I800 anklingt, ${ }^{22}$ sondern mit diesem sich kaum noch ins Gattungsschema der großen Erzählung fügenden Werk hat Goethe den Roman eines individuellen Bildungsganges zur gesellschaftlichen Situation der Moderne geöffnet, zum Maschinenwesen etwa und zum Mythos Amerika. In unserem Zusammenhang interessiert vor allem Lenardos Exkurs über das Auswandern, der mit einem Hinweis auf die Beduinen der Wüste beginnt und auch hernach von einigen Leitvokabeln flankiert wird, die bereits aus dem Karawane-Gedicht bekannt sind. In dieser I82 I niedergeschriebenen Passage heißt es:

»Wir wollen [...] unsere Aufmerksamkeit dem zusammenhängenden, weiten breiten Boden so mancher Länder und Reiche zuwenden. Dort sehen wir große Strecken des Landes von Nomaden durchzogen, deren Städte beweglich, deren lebendig-nährender Heerdenbesitz überall hinzuleiten ist. Wir sehen sie in Mitten der Wüste, auf großen grünen Weideplätzen, wie in erwünschten Häfen vor Anker liegen. Solche Bewegung, solches Wandern wird ihnen zur Gewohnheit, zum Bedürfniß; endlich betrachten sie die Oberfläche der Welt, als wäre sie nicht durch Berge gedämmt, nicht von Flüssen durchzogen. $\ll^{23}$

Die Auswanderer, wie man Goethes Nomaden wohl nennen muss, kommen aus einer Welt, in der die Ökonomie der Territoriale sich verfestigt hat und alles determiniert. »Betrachten wir«, so argumentiert Lenardo, »des festen Landes bewohnteste Provinzen und Reiche, so finden wir überall, wo sich nutzbarer Boden hervortut, denselben bebaut, bepflanzt, geregelt, verschönt und in gleichem Verhältnis gewünscht, in Besitz genommen, befes-

$22 \mid$ »Der Mensch kanns nicht verläugnen, daß er einst glüklich war, wie die Hirsche des Forsts, und nach unzähligen Jahren klimmt noch in uns ein Sehnen nach den Tagen der Urwelt, wo jeder die Erde durchstreifte, wie ein Gott, eh, ich weiß nicht was? den Menschen zahm gemacht, und noch, statt Mauern und todtem Holz, die Seele der Welt, die heilige Luft allgegenwärtig ihn umfieng« (Friedrich Hölderlin: Hyperion oder der Eremit in Griechenland. Sämtliche Werke und Briefe, München I992, Band I, S. 715).

23 I Johann Wolfgang Goethe: Wilhelm Meisters Wanderjahre oder Die Entsagenden. Werke, Band 8 (Hamburger Ausgabe), Io. Aufl., München I98I, S. 385 . 
tigt und verteidigt. ${ }^{24}$ Das Problem an dieser territorialen Eigentumsordnung, so fügt Lenardo unmissverständlich hinzu, sind die zu spät und zu kurz Gekommenen, denn ihrer werden immer mehr. Von »übervölkerten Gegenden « ist die Rede, die ihren Bewohnern keinen Raum mehr lassen, sondern die Konsequenz des Auswanderns nahe legen. »Was wir von Fremden zu erwarten haben, wäre schwer zu sagen; wundersam aber ist es, dass durch eigene Übervölkerung wir uns einander innerlich drängen und, ohne erst abzuwarten, dass wir vertrieben werden, uns selbst vertreiben, das Urteil der Verbannung gegen einander selbst aussprechend. $\ll^{25}$

Wie in allen Migrationsprozessen gibt es auch hier >pull and push «-Faktoren, also einerseits Gründe wie Überbevölkerung, Konkurrenz oder Knappheit, die Leidensdruck erzeugen, zur Emigration drängen und die Auswandernden gleichsam hinausschieben. Auf der anderen Seite aber stehen die verlockenden Reize der Fremde selbst; mobilisiert und angezogen werden die Wanderer durch die zugkräftige Vorstellung eines unerschöpflichen Siedlungsraumes, wie ihn in diesem Falle das zukunftsträchtige Amerika verkörpert. »Hier ist nun Zeit und Ort«, schließt Lenardo sein Plädoyer, »ohne Verdruß und Mißmut in unserm Busen einer gewissen Beweglichkeit Raum zu geben, die ungeduldige Lust nicht zu unterdrücken, die uns antreibt, Platz und Ort zu verändern.«

Zwar geht es noch nicht um Kolonien für ein Volk ohne Raum, wohl aber um die Bereitschaft, seinen eigenen Platz und Ort zu verändern, die als sogar »ungeduldige«, mithin aufgestaute »Lust« eine entsprechende Disposition des Raumes zum Gegenpart hat. Wer in dieser Weise zum Aufbruch drängt, supponiert, dass es irgendwo auf der Welt Regionen gibt, die auf solche Wanderer gewartet haben. Die wahre Zugkraft dieser Beweglichkeit indes ist weder am Ausgangs- noch am prospektiven Zielort zu suchen, sondern im Durchmessen und Überwinden des Raumes selbst. Der Weg vor allem der ungebahnte - ist der beste Treiber; und der stets zurückweichende Horizont, die, wie Koschorke es nennt, »Horizontflucht der romantischen Landschaftsdarstellungen«, ist ein perspektivischer Tantalus-Trick, um diesen Antrieb durch einen Realisierungs-Suspens zu perpetuieren.

Es sind demnach zwei mediale bzw. epistemologische Errungenschaften, die in der Konzeptualisierung des leeren (zu erobernden) Raumes ästhetisch zusammenwirken: erstens die Perspektive (das >Hindurchschauen auf $)$ als Transgressionsraum und Projektionseffekt, zweitens die nun spezifischer an literarischen Darstellungen zu beobachtende Kongruenz von Handlungslinie und durchmessener Raumstrecke, die Robert Musil im Mann ohne Eigenschaften als Sinnstiftung durch den »Faden der Erzählung« beschrieben hat. ${ }^{26}$ Diese Isomorphie von Schrift und Weg, die sich von

24 I J.W. Goethe: Wanderjahre, S. 384.

25 | Ebd., S. 385; das folgende Zitat ebd. S. 386.

26 In dieser kausalen Verknüpfungsleistung sieht Robert Musil (respektive 
den Wanderer-Mythen der Antike und des Mittelalters herleitet und in der Neuzeit in der Figur des Gottsuchers, des Pilgers, des Reisenden schließlich eine textimmanente Position gefunden hat, ${ }^{27}$ inkorporiert den Vorgang der Raumerschließung einem Subjekt der Narration, das - nach dem gleichnamigen Roman J.F. Coopers - als »pathfinder« durch die Widrigkeiten der Außenwelt, aber auch durch das Gewebe des Textes selbst agiert. Indem dieses Text-Ich seinen Weg macht, markiert es den durchquerten Raum: ${ }^{28}$ durch performative Akte des Benennens und Beschreibens, aber auch schon durch die Bewegung selbst.

Die Dynamik des »Zuges« im doppelten Sinne, wie ihn Goethes Wanderjahre und schon das Karawanen-Gedicht entfalten, erfreut sich in späteren Diskursen der Landnahme gewisser Beliebtheit, da der scheinbar naturwüchsige Effekt der Horizontflucht die prekäre Frage nach den Auftraggebern und Adressaten kolonialen Raumgewinns auf unverfängliche, elementar-ästhetische Weise zu beantworten hilft. Wenn man einen Weg vor sich hat oder eine Trasse, einen Flusslauf oder eine Schneise durch den Wald, dann wollen diese Wege mit ihren zum Horizont laufenden Fluchtlinien auch gegangen werden; anders als in dieser unpersönlichen Form kann man es tatsächlich nicht ausdrücken.

Und wo noch kein Weg ist, gilt dies umso mehr. Dafür ist der berühmtberüchtigte »scramble for Africa«, die wohl nachhaltigste koloniale Erfahrung der letzten zweihundert Jahre, ein gutes Beispiel. Afrika ist der letzte und schwierigste Fall für den europäischen Kolonialismus. Warum das so war und wie es zum Mythos des »dark continent « kam, darüber hat bereits Herder einige Vermutungen angestellt. In seinen Ideen zur Philosophie der Geschichte der Menschheit unternimmt er den Versuch, die epochalen kulturellen Prozesse der Menschheitsgeschichte in den Rahmen einer entelechialen Interpretation von Naturgeschichte einzubetten. Untersucht wird hierbei auch, welche natürlichen - etwa astronomischen, geophysischen oder klimatischen - Bedingungen die Existenz menschlichen Lebens und die Herausbildung zivilisatorischer Strukturen befördert haben. Wenig überra-

sein alter ego) eine perspektivische Zurichtung von Geschehnissen durch ihre lineare Erzählform wirksam werden. »Wohl dem, der sagen kann >als<, >eheく und >nachdem««, räsoniert der Mann ohne Eigenschaften, bezeichnenderweise auf seinem Nachhauseweg. »Das ist es, was sich der Roman künstlich zunutze gemacht hat: der Wanderer mag bei strömendem Regen die Landstraße reiten oder bei zwanzig Grad Kälte mit den Füßen im Schnee knirschen, dem Leser wird behaglich zumute« (Robert Musil: Der Mann ohne Eigenschaften, Reinbek 1978, S. 650).

27 | Vgl. Z. Bauman: Vom Pilger zum Touristen.

$28 \mid$ ॥ne of the most enigmatic ways in which writing functions in colonizing is the marking of the earth through the very presence of Europeans moving through the landscape« (J. Noyes: Colonial Space, S. Io9). 
schend kommt Herder dabei zu dem Schluss, dass gerade die Erde mit ihrem eher mittleren Sonnenabstand, auf der Erde wiederum die mittleren, gemäßigten Zonen und unter diesen dann der eher kleine, aber reich mit Binnengliederungen ausgestattete Kontinent Europa die idealen Voraussetzungen mitbringt, derer ein kulturell hoch stehendes Gemeinwesen bedarf. $^{29}$

Aufschlussreich in methodischer Hinsicht ist allerdings die Verbindung, die Herder im I785 erschienenen ersten Buch seiner Ideen zwischen geophysischen Bedingungen und geschichtlichen Prozessen herstellt; ${ }^{30}$ ähnliche Überlegungen zum Einfluss geographischer Faktoren auf die Formen der Sittlichkeit und die kulturellen Differenzen im Leben der Völker hatte zuvor bereits Montesquieu vorgetragen. In der geographischen Grobgliederung, welche »die Natur mit den Bergreihen, die sie zog, wie mit den Strömen, die sie herunter rinnen ließ«, vornahm, habe sie, so Herder, "gleichsam den rohen aber festen Grundriß aller Menschengeschichte [...] entworfen. «I Im Vergleich der geophysischen Struktur der Kontinente glaubte Herder nun die Determinanten der Kolonialgeschichte ablesen zu können. Über Amerika beispielsweise bemerkt er:

»Amerika ist vielleicht auch deswegen voll so viel kleiner Nationen, weil es nord- und südlich mit Flüssen, Seen und Bergen durchschnitten und zerhackt ist. Seiner Lage nach ists von außen das zugangbarste Land, da es aus zwei Halbinseln bestehet, die nur durch einen engen Isthmus zusammenhangen, an dem die tiefe Einbucht noch einen Archipelagus von Inseln bildet. Es ist also gleichsam ganz Ufer; und daher auch der Besitz fast aller Europäischen Seemächte, so wie im Kriege immer wieder Apfel des Spiels. Günstig ist diese Lage für uns europäische Räuber; ungünstig war seine innere Durchschnittenheit für die Bildung der alten Einwohner.« ${ }^{32}$

Im Vergleich zu Amerika, dem zur Kolonisierung scheinbar naturwüchsig prädestinierten Kontinent, sieht Herder Afrika als den Protagonisten der großen Verweigerung unter den Global Players: »Bei Afrika ists offenbar, daß die Sitten seiner Einwohner weniger verschieden sind, weil diese durch keine Meere und Meerbusen, sondern vielleicht nur durch die Wüsten von einander getrennt werden. Auch fremde Nationen haben daher weniger auf dasselbe wirken können und uns, die wir alles durchkrochen haben, ist die-

29 | J.G. Herder: Ideen; zu Europa heißt es dort: »der vielgegliederte kleine Welttheil ward also der Markt und das Gedränge aller Erdvölker im Kleinen« (S. 47).

30 | »Meere, Bergketten und Ströme sind die natürlichsten Abscheidungen so der Länder, so auch der Völker, Lebensarten, Sprachen und Reiche«, sie seien stets »die Direktionslinien oder die Grenzen der Weltgeschichte gewesen« (J.G. Herder: Ideen, S. 45).

31 I Ebd., S. $44 \mathrm{f}$.

32 | Ebd., S. 47. 
ser ungeheure Erdteil so gut als unbekannt.«33 Fast wörtlich ist in diesem Bild des alles durchkriechenden Europa der »scramble for Africa« vorweggenommen. Zwar sieht es aus heutiger Warte zunächst so aus, als habe die Geschichte der letzten zweihundert Jahre Herder Lügen gestraft, denn Afrika ist wesentlich stärker durch die Folgen des europäischen Kolonialismus gezeichnet als die beiden Amerika, die seit Anfang des I9. Jahrhunderts politische Selbstständigkeit erlangt haben. Dennoch kann Herders spekulative These insofern eine gewisse Plausibilität beanspruchen, als die nachhaltige kulturelle Prägung Nord- und Lateinamerikas durch europäische Kolonialnationen zumindest in den zentralafrikanischen Gebieten kein vergleichbares historisches Pendant hat.

\section{Die Suche nach den Nilquellen}

Die geographische Struktur Afrikas erwies sich tatsächlich für eine Erschließung von außen, durch europäische Kolonisatoren, als höchst ungünstig. Der Umriss des Kontinents ist kaum gegliedert, und es gibt nur wenige Inseln, Halbinseln und Meeresbuchten. Hinzu kommt, dass das große Hochlanddreieck des südlichen Afrika von einem Gebirgswall umgeben ist, der wie eine riesige Schüssel an den Außenrändern die höchsten Erhebungen aufweist. So kommt es, dass jenseits einer schmalen Küstenzone der Rand des Hochlandes wie ein Gebirge aufsteigt und den Zugang zum Inneren erschwert. Aber gerade dieser Skandal der hartnäckigen Unerreichbarkeit, Unzugänglichkeit und Undurchdringlichkeit eines riesigen Kontinents stachelte das »colonial desire« im Sinne eines Begehrens der Raumerschließung, des Raumgewinns an. ${ }^{34}$ Wenige Jahre nach Herders Schrift konstituierte sich in England die »African Association« (I788), die sich unter Vorsitz von Joseph Banks dezidiert der Erforschung des Inneren von Afrika verschrieb. ${ }^{35}$ Im Laufe des I9. Jahrhunderts zogen Frankreich und das Deutsche Reich mit ähnlichen Institutionen nach. ${ }^{36}$

33 | Ebd., S. $46 f$.

34 | Zu diesem Konzept vgl. Robert J.C. Young: Colonial Desire. Hybridity in Theory, Culture and Race, London 1995.

35 | Der vollständige Name der Vereinigung lautete »Association for Promoting the Discovery of the Interior Parts of Africa

36 | Die französische »Societé de Géographie« (I82I) und die »Gesellschaft für Erdkunde« (I828) in Berlin gaben ihrerseits wiederum Impulse zur Konstituierung der Londoner »Geographical Society«. Die deutsche »Afrikanische Gesellschaft« (1878) ging aus der »Deutschen Gesellschaft zur Erforschung des äquatorialen Afrika« hervor, die I873 zur Finanzierung der Loango-Expedition gegründet worden war; Initiator war Adolf Bastian, der seit Ende der Goer Jahre den Vorsitz der Berliner »Gesellschaft für Erdkunde« innehatte. 
Das Hauptproblem, das die von der »African Association« initiierten Exkursionen zu gewärtigen hatten, waren die mangelhaften Verbindungen zwischen den von der Seefahrt längst vermessenen Küstenlinien und dem noch weitgehend unbekannten Hinterland. Zwar wiesen die breiten Mündungen großer Flüsse auf die wichtigsten Wasseradern des Kontinents hin, doch stellten diese Ströme den Forschungsreisen und Beutezügen keine so einfachen Zugänge bereit, wie dies etwa in Lateinamerika der Fall war, wo die Hafenstädte an den großen Flussmündungen als europäische Staubsauger fungierten, die den reibungslosen Abtransport der Reichtümer des Hinterlandes gewährleisteten. Die afrikanischen Ströme haben ihre schluchtenreichsten und verkehrsfeindlichsten Abschnitte gerade im Unterlauf, sodass Boote oder andere Lasten oftmals weite Strecken über Land transportiert werden mussten. So blieben Ursprung, Verlauf und zuweilen sogar die Fließrichtung der großen afrikanischen Ströme den Europäern bis ins I9. Jahrhundert hinein ein Mysterium, das zur Legende des dunklen, geheimnisvollen Kontinents beitrug.

Wenn - wie Michael Ondaatje seinen englischen Patienten sagen lässt das I9. Jahrhundert »das Jahrhundert der Flußsucher« war, so hatte das im Falle Afrikas zuvörderst durchaus pragmatische Gründe. Wer den Verlauf und die Schiffbarkeit der großen Ströme bestimmen konnte, hatte den Schlüssel zum ganzen Kontinent. Mit Mungo Parks Exkursionen zum Niger, dem drittgrößten Strom Afrikas, nahm die »African Association« Ende des I8. Jahrhunderts ihre praktische Arbeit auf. Parks Erkundungen des Niger, die der amerikanische Bestsellerautor T.C. Boyle in seinem Abenteurerroman Water Music zu einer neobarocken Version des Herr-und-KnechtThemas verarbeitet hat, bilden zusammen mit der I770 von James Bruce unternommenen Erforschung der äthiopischen Ursprünge des Blauen Nil den Startschuss für die legendären Flusssucher auf dem afrikanischen Kontinent. Und schon an Mungo Parks Vorgehensweise lässt sich erkennen, welche Form des Wissens bei dieser Flusssuche eigentlich auf dem Spiel stand: Es ging um die Suche nach Kohärenz, um das Problem, den lückenlosen Verlauf und Zusammenhang des Stromes nicht nur zu ermitteln, sondern durch eigenen Nachvollzug gleichsam unter Beweis zu stellen.

Die Mündung des Niger war schon den Portugiesen des I6. Jahrhunderts bekannt, und man hatte gleichfalls von einem bei Timbuktu fließenden Strom gehört, diese beiden geographischen Daten aber lange Zeit nicht miteinander in Verbindung gebracht. Vermutet wurde ein Konnex mit dem Gambia, dem Senegal oder dem Nil. Vom Gambia aus drang Mungo Park I795-97 an den mittleren Niger vor und erbrachte durch seine Reise den Nachweis, dass der Niger im Sudan tatsächlich nach Osten fließt, also mit dem Senegal oder gar dem Gambia nichts zu tun hat. Die zweite Initiative zur Rekonstruktion des Niger-Verlaufs war die Expedition Friedrich Konrad 
Hornemanns (I772-I8OI), des ersten deutschen Afrikaforschers, ${ }^{37}$ der seit I796 im Dienst der »African Association« stand. Hornemann sollte von Ägypten aus zum Niger vorstoßen, weil man noch an eine Verbindung mit dem Nil glaubte. I798, während Napoleons Heer von Soldaten und Wissenschaftlern bei den Pyramiden stand, unternahm Hornemann seine »denkwürdige Erstdurchquerung« der Sahara und des Sudan. »Als moslimischer Kaufman verkleidet, schloss er sich einer Karawane an, die über die Oasen Siwa und Audschila auf noch nie von Europäern betretenen Wegen nach Mursuk im Fessan zog. [...] Hornemann, der dauernd fürchten musste, als Christ und Europäer entlarvt zu werden, führte unter schwierigsten Bedingungen seine Beobachtungen durch. Nach einem Abstecher von Mursuk nach Tripolis brach er I800 mit Unterstützung des Paschas in Tripolis von Mursuk nach Bornu auf, um dann weiter zum Niger vorzudringen.« Seit dieser Zeit blieb er verschollen und hinterließ lediglich seine Reiseaufzeichnungen der ersten Teiletappen, die er nach England übersandt hatte. ${ }^{38}$

Während die Expedition von Clapperton das Niger-Problem noch vor I830 definitiv lösen konnte, erwies sich die schon früher begonnene, ja bereits im Altertum diskutierte Suche nach den Quellen des Nil als wesentlich langwieriger und komplizierter. Dass sich die Quelle eines der großen Kulturströme entzieht und verbirgt, ist seit jeher symbolisch gelesen worden; im Grunde präfiguriert sich in diesem Topos schon jener spätere des heart of darkness, des geheimen Herzens eines dunklen Kontinents. Das Rätsel erschien umso größer, als dieser Fluss, der von alters her als längster Strom der Erde galt, in seinem bekannten Verlauf fast keine Nebenflüsse aufweist, aber dennoch gewaltige Wassermassen führt, denen sich die altägyptische Hochkultur verdankte. Ptolemäus und Herodot hatten die Kunde von fernen, schneebedeckten Bergen im Inneren Afrikas kolportiert, den so genannten Mondbergen, welche die Quellen des Nil speisen sollten. An ihm, der sein Haupt (das >caput Nili<) verbarg, fand das Jahrhundert der Flusssucher seinen prominentesten Gegenspieler. Zunächst versuchten die Flussforscher, den Verlauf des Nil von Ägypten und dem Sudan aus fluss-

37 | Ein Schwerpunkt der deutschen Afrikaforscher sollte die Sahara- und Sudanforschung werden, die mit James Richardsons Expedition von I849 begann und mit Heinrich Barths Durchquerung der Wüste und seiner Erforschung der Tschadländer und des Westsudan I850-55 fortgesetzt wurde. Der ihm folgende Eduard Vogel drang 1856 (wie später Gustav Nachtigall) vom Tschad aus nach Wadai vor und fand dort den Tod. Gerhard Rohlfs durchwanderte I86I das Atlasgebirge und durchquerte I865-67 und I869 die Lybische Wüste. Gustav Nachtigall erreichte I869-74 das Saharahochland Tibesti.

38 | Walter Krämer (Hg.): Die Entdeckung und Erforschung der Erde. Mit einem ABC der Entdecker und Forscher, 6. Aufl., Leipzig I970, S. 286; Hornemanns Aufzeichnungen wurden I802 von C. König herausgegeben als Tagebuch seiner Reise von Cairo bis nach Murzuck in den Jahren I797-98. 
aufwärts zu erschließen: W.G. Browne nach Darfur (I793-I796), Burckhardt in Nubien (I8I3-I4), Kinant de Bellefonds (I827) sowie d'Arnaud und Werne am Weißen Nil (I84I). Angestrebt war damit zugleich ein Reiseweg, der einer natürlichen Ader des Kontinents folgen sollte. Dieser Weg entlang des von der Natur vorgezeichneten Flussbettes hatte im Verständnis der Flusssucher nicht die Gewaltsamkeit einer erst willkürlich zu bahnenden Schneise, und er gab den Einheitsvorstellungen Auftrieb, indem er eine lückenlose und permanente Verbindung quer durch den halben Kontinent im Nachvollzug zu zelebrieren unternahm.

Zum Erfolg führte die Suche nach der oder den Quellen freilich erst, als man diese Art von Kontinuitäts-Paradigma aufgab und nicht mehr auf den lückenlosen Verlauf fixiert blieb, sondern einen Quereinstieg suchte. Um die Jahrhundertmitte setzte sich die Idee durch, Nil-Expeditionen nicht vom Unterlauf, sondern von der afrikanischen Ostküste her anzubahnen, wodurch sich die strategische Bedeutung des englischen Stützpunktes auf Sansibar und vor allem die des gegenüberliegenden Festlandhafens Bagamoyo spürbar erhöhte. ${ }^{39}$ Ausschlaggebend für den neuen Forschungsschub in Sachen Nilquelle waren die Berichte zweier deutscher Missionare, die das legendäre Wissen der Antike von den schneebedeckten Mondbergen plötzlich in den Rang einer Tatsache erhoben. Und an dieser Stelle beginnt sich das Thema der Flusssuche mit einem zweiten Szenario zu verbinden, dem der Berge und ihrer Exploration.

Johann Rebmann und Johann Ludwig Krapf wussten Unglaubliches von ihren Reisen zu berichten - sie waren I848 zum Kilimandscharo und I849 zum Mount Kenia vorgedrungen. ${ }^{\circ 0}$ Schneebedeckte Berge im Inneren Afrikas - dies schien so sehr den Fabelberichten der Antike von sagenhaften Mondbergen am Ende der Welt zu gleichen, dass sich diese Neuigkeiten in der geographischen community nur schwer durchsetzen konnten. Aufgrund von detaillierten Befragungen der Eingeborenen gelang Krapf wenig später eine erstaunlich genaue Lokalisierung des Ukerewe-Sees, der seit Speke als Victoriasee firmiert, doch seine und auch Rebmanns Hinwei-

39 I Man kann das übrigens auch an der Konjunktur solch magischer Ortsnamen in literarischen Texten ablesen, die im Falle Bagamoyos in der zweiten Jahrhunderthälfte anhebt und bis in die Moderne reicht, etwa zu Hanns Henny Jahnns Fluß ohne Ufer.

40 | Vom 27. April bis Io. Juni I848 unternahm Johann Rebmann eine Fahrt in das Dschagga-Land. Am II. Mai I848 erblickte er erstmals den höchsten Berg Afrikas. »Wir sahen diesen Morgen die Berge von Dschagga immer deutlicher, bis ich gegen to Uhr den Gipfel von einem derselben, mit einer auffallend weißen Wolke bedeckt, zu sehen glaubte. Mein Führer hieß das Weiße, das ich sah, schlechtweg >Kälte< (beredi); es wurde mir aber eben so klar als gewiß, daß das nichts anderes sein könne als Schnee« (Johann Ludwig Krapf: Reise in Ostafrika I837-I855, hg. von Hanno Beck, 2 Bde., Stuttgart I964, Band II, S. 3of.). 
se auf gewaltige Binnenseen, die dann in >Petermanns Mitteilungen< publiziert wurden, ${ }^{4 \mathrm{I}}$ stießen auf Skepsis; so trat etwa der englische Geograph William Desborough Cooley ${ }^{42}$ im selben Organ mit einer energischen Gegendarstellung hervor. ${ }^{43}$ Der Berliner Geograph Carl Ritter indes würdigte ausdrücklich den Anteil, den die beiden Missionare an der Erforschung Afrikas hatten, und auch der Nestor der deutschen Geographie, Alexander von Humboldt, verfolgte interessiert die Diskussionen, die durch die >Entdeckungen < des Kilimandscharo und Mount Kenia ausgelöst wurden. ${ }^{44}$

Die Suche nach der oder den Nilquellen wurde nun hauptsächlich von englischen Initiativen vorangetrieben. Da dieses meist als heroische Phase der Afrika-Geographie erzählte Kapitel des Wettlaufs zu den Nilquellen weitgehend bekannt ist, ${ }^{45}$ braucht es hier nur stichwortartig rekapituliert zu werden. Burton und Speke waren die Ersten, die nicht dem Flusslauf folgten, sondern besagten Quereinstieg über Sansibar wählten und so relativ rasch in das zentrale Hochlandgebiet vordringen konnten. I858 entdeckten sie zusammen den Tanganjikasee, und Speke stieß auf den Victoriasee. In einer genialischen Extrapolation mutmaßte Speke sofort, beim Ausfluss dieses gewaltigen Wasserreservoirs müsse es sich um die gesuchte Nilquelle handeln, und nach dem Dissens mit Burton verbreitete er seine These im Alleingang. Bei einer zweiten Expedition I86o-63 folgte er diesem Wasserlauf nach Norden, während Baker, von Norden kommend, den vom Nil durchflossenen Albertsee entdeckte.

Ein riesiges Stück des fehlenden Flussverlaufs konnte damit rekonstruiert werden. Livingstone erweiterte dieses Wissen mit seinen Reisen zum Tanganjikasee (I871/72), was schließlich zu seiner spektakulären >Rettung< durch Stanley führte - bekanntlich ein Coup jener Zeitungen, die Stanleys Hilfsexpedition finanziert hatten. Stanley, sein Retter und Nachfolger, setz-

41 | Zu Rebmanns Briefen und seiner Karte, die »das Herz Afrikas« enthüllen sollte, vgl. August Petermann: »Ukerewe, das große Binnenmeer von Inner-Afrika«, in: Petermanns Geographische Mitteilungen (I855), S. $233 \mathrm{f}$.

42 | Cooley ging noch 1864 davon aus, dass der Njassa- und der TanganjikaSee ein großes Binnengewässer bildeten (vgl. Hanno Beck: Große Reisende. Entdecker und Erforscher unserer Welt, München I97I, S. 225).

43 | 856 gab Petermann eine ergänzte Karte Rebmanns heraus und ließ auch die Gegenposition Cooleys zu Wort kommen; vgl. »J. Erhard's Mémoire zur Erläuterung der von ihm und J. Rebmann zusammengestellten Karte von Ost- und CentralAfrika. Nebst Bemerkungen von W. Desborough Cooley und A. Petermann«, in: Petermanns Geographische Mitteilungen (1856), S. I9-32; vgl. ebd. (I864), S. 449-456.

44 | Vgl. Alexander von Humboldt: Briefe an Christian Carl Josias Freiherr von Bunsen, Leipzig I869, S. I25f. und S. I73.

45 | Vgl. dazu Georg Brunold (Hg.): Nilfieber. Der Wettlauf zu den Quellen. Mit Beiträgen von Alan Moorehead, James Bruce, Richard Francis Burton, David Livingstone, Henry Morton Stanley und anderen, Frankfurt am Main I993. 
te die Quellensuche dort fort, wo schon Speke eine definitive Antwort gefunden zu haben glaubte, am Victoriasee. Gegen Spekes These ließ sich ja in der Tat einwenden, dass Flüsse für gewöhnlich nicht in Seen entspringen, sondern im Gebirge. Stanley gelang es in der Folge, als wichtigsten $\mathrm{Zu}$ fluss des Victoriasees den Kagera zu ermitteln, und somit erwies sich jenes berühmte Telegramm Spekes an die »Royal Geographical Society« als voreilig, in dem er triumphiert hatte: »The Nile is settled!« Quasi >nebenbei< erschlossen all diese Exkursionen auch das bei der Anreise durchquerte Küstengebiet; bemerkenswert erscheint dabei insbesondere, dass die von den Expeditionstrecks gewählte Route genau die Linienführung der späteren Trasse der »Tanganyika Railway beschreibt. $^{46}$

In den Jahren I892/93 unternahm der Österreicher Oscar Baumann eine Exkursion, die zunächst ins Massailand, dann zum Victoriasee und schließlich ins Einzugsgebiet des Kagera führte. Ausgerüstet wurde seine Expedition vom »Deutschen Antisklaverei-Komitee«, das unter Vorsitz des Fürsten Wilhelm zu Wied stand; zu den Auftraggebern gehörten ferner die »Deutsch-Ostafrikanische Gesellschaft« und die »Eisenbahn-Gesellschaft für Deutsch-Ostafrika«, denn Baumanns Mission bestand unter anderem darin, von Tanga aus eine möglichst kurze Verbindung zum Victoriasee zu erkunden, die für einen Bahnbau geeignet wäre. Bereits I888 hatte Baumann zusammen mit dem Leipziger Kolonialgeographen Hans Meyer eine Reise durch Ostafrika unternommen und die Usambara-Region erkundet, wo ein ausbrechender Araberaufstand weitere Forschungen verhinderte (insbesondere die geplante Erstbesteigung des Kilimandscharo, die Meyer dann im folgenden Jahr gelingen sollte). Die Reise von I892/93, über die Baumann in seinem Buch Durch Massailand zur Nilquelle (1894) berichtet, verbindet den Einsatz für das Anti-Sklaverei-Komitee mit jenem für den Eisenbahnbau. Ausdrücklich dankt Baumann in seinem Reisebericht jenen, die von anderen Forschungsreisenden regelmäßig »mit vornehmem Stillschweigen übergangen zu werden« pflegten: den schwarzen Trägern und Soldaten seiner Expedition, die über Monate seine einzigen Gefährten waren. ${ }^{47}$

Was die Frage der Nilquellen betrifft, so konnte Baumann den von Stanley als wichtigsten Zufluss des Victoriasees ermittelten Kagera bis in sein Quellgebiet zurückverfolgen; als erster Europäer drang er nach Ruanda vor und gelangte auf seiner Rückreise zum Nordende des Tanganjikasees. Baumann brachte kartographische Aufnahmen, geographische und völkerkundliche Studien mit, die er auf weiteren Expeditionen in Usambara und auf den Küsteninseln ergänzte. Nun sei sicher, resümiert er in seinem Be-

46 | G. Brunold: Nilfieber, S. 22.

47 | Oscar Baumann: Durch Massailand zur Nilquelle. Reisen und Erforschungen der Massai-Expedition des deutschen Antisklaverei-Komite in den Jahren I89II893, Berlin I894, S. VIII. 
richt, dass »die letzten Schleier« des Nilproblems »gelüftet wurden« und »dass das >Caput Nili Quaerere< von nun an endgiltig der Vergangenheit angehört«.$^{48} \mathrm{Ab}$ I896 war Baumann österreichischer Konsul in Sansibar, erlag aber schon Ende I899 einer Infektionskrankheit. Seine Behauptung, die Nilquellenfrage definitiv gelöst zu haben, blieb naturgemäß nicht unwidersprochen. Im Jahre I904 erschien Richard Kandts Reisebericht Caput Nili, eine kritische Auseinandersetzung mit Baumanns zum Teil fehlerhaften Übersetzungen und Zuschreibungen, vor allem aber mit seiner These, die Hauptquelle des Nil gefunden zu haben. Auch für Kandt führte die Spur nach Ruanda, und sein Reisebericht enthält die ersten ausführlichen Nachrichten über dieses Land, seine Ethnien und seine geographische Beschaffenheit.

Kandt hatte seine I 897 begonnene, mehrjährige Forschungsreise selbst finanziert und war ohne Auftraggeber, wohl aber mit Billigung und Unterstützung der Kolonialverwaltung von Deutsch-Ostafrika unterwegs; nach deren erfolgreichem Abschluss wurde ihm I907 die Funktion des kaiserlichen Residenten in Ruanda übertragen. In seinem Bericht herrscht weder Chronologie noch Kontinuität des Schauplatzes, sondern eine fast aleatorische Mixtur aus Rückblenden, Tagebucheintragungen im Gegenwartsmodus und eingestreuten Exkursen, die der Autor schlichtweg als Sammlung von »Briefen« bezeichnet.

Die Disparität dieser Textelemente lässt eine Spannung erkennen zwischen dem protokolonialen Entdeckerfieber - jenem in der langen Geschichte geographischer Exploration so zählebigen Wunsch, »der Erste zu sein « - und der pragmatischen Forderung, innerhalb des kolonialen Terrains bestimmte Formen der Infrastruktur zu etablieren, die nicht auf Originalität, sondern auf Funktionalität ausgerichtet sein mussten. Im Rückblick Kandts auf das eigene Werk wird diese Spannung als ein Vorher/ Nachher-Doppelbild in Szene gesetzt. »Als dies Buch geschrieben wurde, gab es keine 50 Kilometer Bahn in Ostafrika, heute 2000, betrug der Handel keine I5 Millionen, heute über 80, lebten 1500 Europäer dort, heute über 6000, brauchte ein Brief zu mir nach Ruanda 3 Monate, heute 5 Wochen «, 49 resümiert der Autor im Vorwort zur dritten Auflage, das im Juli I9I4 entstand, dem letztmöglichen Zeitpunkt für solche melancholischen Erfolgsbilanzen. Ein prosperierendes, reibungslos funktionierendes deutsches Kolonialwesen, so die Botschaft dieses Rückblicks, habe jenen Pioniergeist, aus dem es einst hervorgegangen sei, weitgehend zum Verschwinden gebracht.

48 I O. Baumann: Durch Massailand, S. I48; vgl. auch S. I28, I45. Dieses Zitat findet sich auch bei Richard Kandt (Caput Nili. Eine empfindsame Reise zu den Quellen des Nils, 6. Aufl., Berlin I92I, S. 4), dem jenes apodiktische Fazit seines Vorgängers jedoch unhaltbar zu sein schien.

49 | Kandt: Caput Nili, XIV. 


\section{Trassen für die Eisenbahn}

Gradmesser und Ausdruck dieser Desillusionierung ist insbesondere das die individuellen Pionierleistungen gleichsam absorbierende Vordringen der Eisenbahn. Die Eisenbahnbauer waren, wie der Fall Deutsch-Ostafrikas belegt, die legitimen Nachfolger der Pfadfinder, Spurensucher und Wegebahner. Auf eben den Routen, auf welchen zunächst die Flusssucher und Bergbesteiger das Land durchquerten, wird sich ab dem Ende des I9. Jahrhunderts dann die Eisenbahn fortbewegen. Die Baumaßnahmen in den deutschen Schutzgebieten wurden unter Federführung der Großbanken begonnen und hauptsächlich von dem Bauunternehmen Philipp Holzmann ausgeführt, das heißt vor allem: durch deutsche Ingenieure überwacht. In den britischen Kolonialgebieten hatten die Erschließungen für Eisenbahntrassen bereits etwas früher begonnen, und auch hier standen die Planungen in unmittelbarer Kontinuität zur Terrainsondierung durch die Nilquellensucher. So hatte sich aus den Expeditionen rund um den Victoriasee eine englische Prädominanz ergeben, die 1896 in der Proklamation des Protektorats Uganda ihren Ausdruck fand. Fünf Jahre später standen die Arbeiten an der Uganda-Bahn von Mombasa nach Kampala bereits vor dem Abschluss. Winston Churchill, damals Unterstaatssekretär für die Kolonien, pries sie hymnisch als »Perle Afrikas«, auf die es alle Kräfte zu konzentrieren gelte.

Die menschlichen Pfadfinder >spurten < für das technische Vehikel. Das war auch in Deutsch-Ostafrika so, wo in den goer Jahren zwei Eisenbahntrassen in Angriff genommen wurden. I89I hatte die »Eisenbahn-Gesellschaft für Deutsch-Ostafrika« die Konzession zum Bau einer Linie von Tanga nach Korogwe erhalten, also von der Küste ins Hochland, der klassischen kolonialen Erschließungsrichtung folgend (und fast parallel zur nicht weit entfernten britischen Linie Mombasa-Nairobi weiter nördlich). Dreizehn Jahre später erreichte die wegen ihrer Streckenführung auch Usambarabahn genannte Trasse Moschi am Kilimandscharo und damit ihr Zielgebiet. Mit dem Bau einer zweiten Linie wurde I905 begonnen; sie folgte der Route, die von den Nilquellensuchern Baumann und Kandt eingeschlagen worden war. Die Bahn sollte von Daressalam, dem etwas südlich von Bagamoyo gelegenen Regierungssitz Deutsch-Ostafrikas, über Tabora bis an den Tanganjikasee führen. Bereits I9II waren zwei Drittel der Strecke fertig gestellt, und I9I4 erreichte man Udjidji am Ufer des Tanganjika.

Aus der Sicht der europäischen Kolonialnationen ließen die geographischen Gegebenheiten in Afrika die Erschließung des Hinterlandes durch Eisenbahnen als besonders geeignet erscheinen. Auf den alten Karawanenpfaden konnte Personen- und Güterverkehr nur mit Trägern durchgeführt werden, was zeitraubend und kostspielig war. Bestimmend war bei diesen Projekten die Zielvorstellung einer möglichst direkten Streckenführung, die auf vorhandene Siedlungsstrukturen und soziale Gegebenheiten vermeint- 
lich keine Rücksicht zu nehmen brauchte. Die Einrichtung eines hindernislosen und reibungsfreien Transportverkehrs mithilfe eines technisch-institutionellen Beförderungsmittels schuf die bis dato effektivste Form der Territorialkontrolle. Verheerend waren bereits die Folgen der Bauphase unter den einheimischen Arbeitskräften..$^{\circ}$ Die meistens zwangsrekrutierte afrikanische Belegschaft wurde während der Bauarbeiten durch harte Arbeitsbedingungen und ausbrechende Krankheiten, hauptsächlich Malaria-Epidemien und Magen-Darm-Infektionen, enorm dezimiert. ${ }^{5 \mathrm{I}}$

Entscheidend aber war der Schlag, den die Eisenbahn gegen das jahrhundertealte Karawanen-Prinzip führen sollte. Richard Kandts Caput Nili kann aus dieser Sicht als ein leidenschaftliches Plädoyer gegen die nomadischen Verkehrsformen gelesen werden. Von der afrikanischen Bevölkerung spricht Kandt als von jenem »Menschenmaterial«, »das in ständiger Bewegung zwischen dem Meere und den großen Seen die Landstraßen frequentiert und in den wenigen Städten von Küste und Hinterland sich zu größeren Mengen anhäuft «..$^{2}$ »Jeder anderen Arbeit als dem Lastentragen abhold, bilden sie eine im Grunde sozial minderwertige Gesellschaft von Menschen, die in Ländern mit härterem struggle for life dem Untergang geweiht wären und zu einem afrikanischen Proletariat sich entwickeln würden, sobald ihnen die Lasten abgenommen und auf den eisernen Rücken von Dampfwagen gelegt würden.«33

Mit dem Einsatz der Technik war dieser Daseinskampf entschieden; die Eisenbahntrasse hatte Lastenträger, reisende Händler und Kundschafter überflüssig gemacht. Das geschmeidige Gleiten auf eisernen Schienen führt, wie es die Pfadfinder in den scheinbar herrenlosen Gebieten der afrikanischen Weite bereits imaginiert hatten, durch ein Land ohne Menschen, durch einen Raum ohne Volk.

50 | Viele der afrikanischen Bauarbeiter waren unfreiwillig zum Bahnbau abgestellt worden und, wie es in einem Bericht über den »Gesundheitsdienst beim Bahnbau« von I907 heißt, »durch die kriegerischen Unruhen und Hungersnöte sehr heruntergekommen« (zit. n. Wolfgang U. Eckart: Medizin und Kolonialimperialismus. Deutschland I884-I945, Paderborn u.a. I997, S. 35I).

51 I Die beiden deutschen Eisenbahnbauprojekte in Ostafrika verzeichneten allein zwischen I909 und I9I2 mehr als tausend Todesfälle unter der afrikanischen Arbeiterschaft. »Eine solche Zahl wäre im Reich selbst bei vergleichbaren Bauprojekten undenkbar gewesen und hätte mit Sicherheit zur Einstellung solcher Vorhaben, wenn nicht zu Skandalen im Reichstag geführt« (Eckart: Medizin, S. 354).

52 | Kandt: Caput Nili, S. II9.

53 | Kandt: Caput Nili, S. I20. 